\title{
Unified Experiment Design, Bayesian Minimum Risk and Convex Projection Regularization Method for Enhanced Remote Sensing Imaging
}

\author{
Yuriy Shkvarko, Jose Tuxpan, and Stewart Santos \\ Department of Electrical Engineering, CINVESTAV-IPN, Guadalajara, Mexico \\ \{shkvarko, jtuxpan, ssantos\} @gdl. cinvestav.mx
}

\begin{abstract}
We address new approach for enhanced multi-sensor imaging in uncertain remote sensing (RS) operational scenarios. Our approach is based on incorporating the projections onto convex solution sets (POCS) into the descriptive experiment design regularization (DEDR) and fused Bayesian regularization (FBR) methods to enhance the robustness and convergence of the overall unified DEDR/FBR-POCS procedure for enhanced RS imaging. Computer simulation examples are reported to illustrate the efficiency and improved operational performances of the proposed unified DEDR/FBR-POCS imaging techniques in the extremely uncertain RS operational scenarios.
\end{abstract}

Keywords: Convex sets, descriptive regularization, experiment design, multisensor imaging, remote sensing.

\section{Introduction}

In this study, we propose a unification of the previously developed descriptive experiment design regularization (DEDR) [1] and the fused Bayesian regularization (FBR) [2] methods for enhanced imaging in the remote sensing (RS) operational scenarios with model uncertainties. The operational uncertainties are associated with the unknown statistics of random perturbations of the signal formation operator (SFO) in the turbulent medium, imperfect sensor system calibration, finite dimensionality of measurements, multiplicative signal-dependent speckle noise, uncontrolled antenna vibrations and random carrier trajectory deviations in the case of SAR. The general DEDR method for solving such class of uncertain RS inverse problems has been constructed in our previous study [3] as an extension of the statistically optimal maximum likelihood (ML) technique [1], in which the spatial spectrum pattern (SSP) estimation error was minimized in a descriptively balanced fashion via weighted maximization of spatial resolution over minimization of resulting noise energy algorithmically coupled with the worst-case statistical performance optimization-based convex regularization. In this paper, we are focused on the design of the unified DEDR/FBR method employing the idea of incorporating the projections onto convex solution sets (POCS) into the corresponding DEDR/FBR-related solution operators to enforce the robustness and convergence. The crucial practical issue relates to proper 
adjustment of the regularization parameters in the unified DEDR/FBR-POCS iterative reconstructive technique to the particular uncertain RS operational scenario. The advantage in using the developed method over the previously proposed RS imaging and de-speckling techniques is demonstrated through the reported computer simulation experiments performed using the elaborated virtual remote sensing laboratory (VRSL) software.

\section{Problem Formalism}

Referring to our previous studies [1]-[3], the random signal $u$ at the output of the sensor system antenna (SAR system in this particular study) moved by the carrier along the deviated linear trajectory $\boldsymbol{\rho}(t)$ in the time instance $t$ relates to the field $e$ scattered from the probing surface through the integral equation of observation

$$
u(\mathbf{p})=(\widetilde{\mathbf{S}} e(\mathbf{r}))(\mathbf{p})+n(\mathbf{p})=\int_{R} \tilde{S}(\mathbf{p}, \mathbf{r}) e(\mathbf{r}) d \mathbf{r}+n(\mathbf{p})
$$

where $\mathbf{p}=(t, \boldsymbol{\rho}(t))$ defines the time-space trajectory points, the complex scattering function $e(\mathbf{r})$ represents the random scene reflectivity over the probing surface in the plane of the scanned scene [6]; $\mathbf{r}$ is a vector of the scan parameters, usually the polar, cylindrical or Cartesian coordinates of the probing surface; the uncertain SFO $\tilde{\mathbf{S}}$ is defined by the integral at the right hand of (1) with the nominal kernel $S(\mathbf{p}, \mathbf{r})=\langle\tilde{S}(\mathbf{p}, \mathbf{r})\rangle$ specified by the time-space modulation of signals employed in a particular imaging SAR system [4]. The variations about the mean $\delta S(\mathbf{p}, \mathbf{r})=\tilde{S}(\mathbf{p}, \mathbf{r})-S(\mathbf{p}, \mathbf{r})$ pertain to the random perturbation component in the SFO.

The spatial spectrum pattern $(\mathrm{SSP}) \quad b(\mathbf{r})=\left\langle|e(\mathbf{r})|^{2}\right\rangle$ represents the ensemble average of the squared modulus of the random complex scene reflectivity $e(\mathbf{r})$ as a function over the analysis domain $R \ni \mathbf{r}$ and is referred to as a desired RS image to be reconstructed from the measurement data recordings. The vector-form model of (1) is given by discrete-form equation of observation (EO) [3]

$$
\mathbf{u}=\widetilde{\mathbf{S e}}+\mathbf{n}=\mathbf{S e}+\Delta \mathbf{e}+\mathbf{n},
$$

where $\mathbf{u}, \mathbf{n}$ and $\mathbf{e}$ define the vectors composed of the coefficients $\left\{u_{m}\right\},\left\{n_{m}\right\}$ and $\left\{e_{k}\right\}$ of the discrete-form approximations of the fields $u, n$ and $e$ with respect to the selected orthogonal decomposition function set $\left\{h_{m}(\mathbf{p})\right\}$ in the observation domain and the pixel set $\left\{g_{k}(\mathbf{r})\right\}$ in the scene domain, respectively [3]. The matrix-form representation of the uncertain SFO in (2) was formalized in [3] by

$$
\tilde{\mathbf{S}}=\mathbf{S}+\Delta
$$

The $M \times K$ nominal SFO matrix $\mathbf{S}$ in (2), (3) is composed of the scalar products $\left\{\left[S g_{k}, h_{m}\right]_{\mathrm{U}}\right\}[1]$, while all problem model uncertainties are attributed to the distortion term $\Delta$. We refer to our previous study [3], where the distortions in the random medium were explained based on the propagation theory models [6]. Note that in practice, one cannot attribute the exact portion of the composite SFO perturbation term $\Delta$ 
to a particular source of disturbances, thus cannot separate in (3) the uncertainties caused by the turbulent medium effects, speckle noise or the observation mismatch errors as those are randomly mixed in the $\boldsymbol{\Delta}$. These practical aspects motivated our adopting in [3] the robust statistical treatment of the irregular SFO perturbations $\boldsymbol{\Delta}$ as a random zero-mean matrix with the bounded second-order moment, i.e.

$$
\langle\Delta\rangle=\mathbf{0} ; \quad\left\langle\|\Delta\|^{2}\right\rangle=\left\langle\operatorname{tr}\left\{\Delta \Delta^{+}\right\}\right\rangle \leq \eta
$$

where $\|\Delta\|^{2}=\operatorname{tr}\left\{\Delta \Delta^{+}\right\}$defines the squared matrix norm, $\operatorname{tr}\{\cdot\}$ is the trace operator, superscript ${ }^{+}$defines the Hermitian conjugate (conjugate transpose), and $\eta$ is the bounding constant [3].

Because of an incoherent nature of the scattering function $e(\mathbf{r})$, vector $\mathbf{e}$ in the equation of observation (2) is characterized by a diagonal correlation matrix, $\mathbf{R}_{\mathbf{e}}=\operatorname{diag}(\mathbf{b})=\mathbf{D}(\mathbf{b})$, in which the $K \times 1$ vector $\mathbf{b}$ of the principal diagonal (composed of the elements $\left.b_{k}=\left\langle\left|e_{k}\right|^{2}\right\rangle ; k=1, \ldots, K\right)$ is referred to as the vector-form SSP. The problem that we solved in our previous studies [1]-[3] was to derive an estimate $\hat{\mathbf{b}}$ of the SSP vector and to reconstruct the desired SSP distribution

$$
\hat{b}_{(K)}(\mathbf{r})=\sum_{k=1}^{K} \hat{b}_{k} g_{k}(\mathbf{r})
$$

over the pixel-formatted observation scene $R \ni \mathbf{r}$ by processing the data vector $\mathbf{u}$ (in the operational scenario with the single processed uncertain data realization) or $J>1$ whatever available recorded independent realizations $\left\{\mathbf{u}_{(j)} ; j=1, \ldots, J\right\}$ of the data (in the scenario with multiple observations) collected with a particular system operating in the uncertain RS environment.

\section{Phenomenology}

\subsection{DEDR Method}

To alleviate the ill-posedness of the SSP reconstruction problem (5) with the uncertain observation model (2)-(4), the DEDR method was constructed in [3] given by

$$
\hat{\mathbf{b}}_{D E D R}=\left\{\mathbf{F}_{D E D R} \mathbf{Y} \mathbf{F}_{D E D R}^{+}\right\}_{\text {diag }}=\left\{\mathbf{K S}^{+} \mathbf{R}_{\Sigma}^{-1} \mathbf{Y} \mathbf{R}_{\Sigma}^{-1} \mathbf{S K}\right\}_{\text {diag }}
$$

that estimates the SSP vector $\hat{\mathbf{b}}$ via applying the DEDR-optimal solution operator

$$
\mathbf{F}_{D E D R}=\mathbf{K S}^{+} \mathbf{R}_{\Sigma}^{-1}
$$

to the data matrix $\mathbf{Y}$ composed of the uncertain data measurements, i.e. the rank-1 (ill-conditioned) outer product matrix $\quad \mathbf{Y}=\mathbf{Y}_{(\operatorname{rank}-1)}=\mathbf{u u}^{+} \quad$ in the scenario with the single recorded data realization (e.g., single-look imaging SAR applications), and the rank- $J$ empirical estimated correlation matrix $\mathbf{Y}=\mathbf{Y}_{(\text {rank-J) }}=(1 / J) \sum_{j=1}^{J} \mathbf{u}_{(j)} \mathbf{u}_{(j)}^{+}$in the scenario with $J>1$ independent multiple observations [3] 
The $\mathbf{S}^{+}$in the solution operator (7) represents the adjoint (Hermitian conjugate [5]) to the nominal SFO matrix $\mathbf{S}$, and $\mathbf{R}_{\Sigma}^{-1}$ is the inverse of the augmented (diagonal loaded) noise correlation matrix defined by [3], $\mathbf{R}_{\Sigma}=\mathbf{R}_{\Sigma}(\beta)=\left(\mathbf{R}_{\mathbf{n}}+\beta \mathbf{I}\right)$. In the practical RS scenarios [4], [5], (and specifically, in the SAR imaging applications), it is a common practice to accept the robust white additive noise model, i.e. $\mathbf{R}_{\mathbf{n}}=N_{0} \mathbf{I}$, attributing the unknown correlated noise component as well as the speckle to the composite uncertain noise term $\Delta \mathbf{e}$ in (2), in which case $\mathbf{R}_{\Sigma}=N_{\Sigma} \mathbf{I}, N_{\Sigma}=N_{0}+\beta$ with the composite noise variance $N_{\Sigma}=N_{0}+\beta$, the initial $N_{0}$ augmented by the loading factor $\beta=\eta \eta / \alpha \geq 0$ adjusted to the regularization parameter $\alpha$, the Loewner ordering factor $\gamma>0$, and to the SFO uncertainty bound $\eta \geq\left\langle\operatorname{tr}\left\{\Delta \boldsymbol{\Delta}^{+}\right\}\right\rangle$(see [3] for details).

Next, we refer to [3] for specifying the family of the DEDR-related estimators for the considered there feasible adjustments of the processing-level degrees of freedom $\left\{\alpha, N_{\Sigma}, \mathbf{A}\right\}$

$$
\hat{\mathbf{b}}^{(p)}=\left\{\mathbf{F}^{(p)} \mathbf{Y} \mathbf{F}^{(p)+}\right\}_{\text {diag }} ; \quad p=1, \ldots, P,
$$

where different employed solution operators $\left\{\mathbf{F}^{(p)} ; p=1, \ldots, P\right\}$ specify the corresponding DEDR-related estimators.

\subsection{FBR Method}

The estimator that produces the high-resolution optimal (in the sense of the Bayesian minimum risk strategy) estimate $\hat{\mathbf{b}}$ of the SSP vector via processing the $M$ dimensional data recordings $\mathbf{u}$ applying the fused Bayesian-regularization (FBR) estimation strategy that incorporates nontrivial a priori geometrical and projectiontype model information was developed in [1], [2]. The FBR method [1], [2] implies two-stage data processing. First, the vector of sufficient statistics (SS) is formed $\mathbf{v}=\left\{\mathbf{F}_{F B R} \mathbf{u u}^{+} \mathbf{F}_{F B R}^{+}\right\}_{\text {diag }}$ applying the regularized solution operator

$$
\mathbf{F}_{F B R}=\mathbf{F}^{(6)}=\left(\mathbf{S}^{+} \mathbf{R}_{\mathbf{n}}^{-1} \mathbf{S}+\hat{\mathbf{D}}^{-1}\right)^{-1} \mathbf{S}^{+} \mathbf{R}_{\mathbf{n}}^{-1}
$$

to the sampled trajectory data signal $\mathbf{u}$. Second, the smoothing window $\mathbf{W}$ is applied to such SS to satisfy the regularizing consistency and metrics constraints [1], [2] that yields the resulting FBR estimator

$$
\hat{\mathbf{b}}_{F B R}=\mathbf{W v}=\mathbf{W}\left\{\mathbf{F}_{F B R} \mathbf{u u ^ { + }} \mathbf{F}^{+}{ }_{F B R}\right\}_{\text {diag }} .
$$

Thus, the FBR method may also be viewed as a particular member of the unified DEDR-related family (8), in which the additional pseudo averaging is performed applying the regularizing window $\mathbf{W}$. 


\section{POCS Regularized Unified DEDR/FBR Technique}

To precede from the general-form DEDR and FBR estimators to the practically realizable SAR-adapted SSP reconstruction techniques, we follow the convex regularization paradigm invoked from the fundamental theorem of POCS [5]. Our approach incorporates the intrinsic factorization and sparseness properties of the SAR ambiguity functions [4], [7] into the construction of the POCS-regularized fixed-point iterative SSP reconstruction procedures that drastically reduces the overall computational load of the resulting algorithms.

To convert the general-from estimators (6) and (10) with the ML-optimally specified degrees of freedom [3] (i.e., $\left.\alpha \mathbf{A}=\mathbf{D}(\hat{\mathbf{b}}), N_{\Sigma}=N_{0}+\beta\right)$ to a unified POCS-regularized fixed-point iterative algorithm, we first, define a sequence of estimates $\left\{\hat{\mathbf{b}}_{[i]}\right\}$ as

$$
\hat{\mathbf{b}}_{[i]}=\mathbf{P}\left\{\mathbf{K}_{[i]} \mathbf{S}^{+} \mathbf{Y S K} \mathbf{S i}_{[i]}\right\}_{\text {diag }}
$$

$i=0,1, \ldots$, where $\mathbf{P}$ is a convergence enforcing projector (in our case, the POCSregularizing operator) [5];

$$
\mathbf{K}_{[i]}=\mathbf{K}\left(\hat{\mathbf{b}}_{[i]}\right)=\left(\Psi+N_{\Sigma} \mathbf{D}^{-1}\left(\hat{\mathbf{b}}_{[i]}\right)\right)^{-1}
$$

represents the self-adjoint reconstruction operator at the $i$ th iteration step and

$$
\Psi=\mathbf{S}^{+} \mathbf{S}
$$

is the nominal system point spread function (PSF) operator [2]. Applying routinely the fixed-point technique [5] to the equation (12), we next, construct the unified POCS-regularized iterative SSP estimation algorithm

$$
\hat{\mathbf{b}}_{[i+1]}=\mathbf{P} \hat{\mathbf{b}}_{[0]}+\mathbf{P} \mathbf{T}_{[i]} \hat{\mathbf{b}}_{[i]} ; i=0,1, \ldots
$$

Here,

$$
\mathbf{T}_{[i]}=\mathbf{T}_{[i]}\left(\hat{\mathbf{b}}_{[i]}\right)=2 \operatorname{diag}\left(\left\{\boldsymbol{\Omega}_{[i]}\left(\hat{\mathbf{b}}_{[i]}\right)\right\}_{\mathrm{diag}}\right)-\mathbf{H}_{[i]}\left(\hat{\mathbf{b}}_{[i]}\right) ; i=0,1, \ldots
$$

represents the solution-dependent matrix-form iteration operator, where

$$
\begin{aligned}
& \boldsymbol{\Omega}_{[i]}=\boldsymbol{\Omega}_{[i]}\left(\hat{\mathbf{b}}_{[i]}\right)=\mathbf{I}-\boldsymbol{\Psi}-N_{\Sigma} \mathbf{D}^{-1}\left(\hat{\mathbf{b}}_{[i]}\right) ; \\
& \mathbf{H}_{[i]}=\mathbf{H}_{[i]}\left(\hat{\mathbf{b}}_{[i]}\right)=\boldsymbol{\Omega}_{[i]}\left(\hat{\mathbf{b}}_{[i]}\right) \circ \boldsymbol{\Omega}_{[i]}^{*}\left(\hat{\mathbf{b}}_{[i]}\right) ;
\end{aligned}
$$

$\circ$ denotes the Shur-Hadamar (element-by-element) matrix product, and the zero-step iteration

$$
\hat{\mathbf{b}}_{[0]}=\hat{\mathbf{b}}_{M S F}=\left\{\mathbf{S}^{+} \mathbf{Y S}\right\}_{\text {diag }}
$$

is formed as an outcome of the conventional matched spatial filtering (MSE) algorithm from the DEDR family (8) specified for the adjoint SFO solution operator $\mathbf{S}^{+}$. The principal advantage of the fixed-point procedure (14) relates to the exclusion of the solution-dependent operator inversions (12), which are now performed in an 
indirect iterative fashion. This transforms the computationally extremely intensive general-form procedures (6), (10) into the iterative technique (14) executable in (near) real computational time.

\section{Simulations}

Having established the unified POCS-regularized DEDR/FBR-related iterative technique (14) for SSP reconstruction, in this section, we present the results of comparative numerical simulations of six different SSP formation/reconstruction algorithms, in particular, the conventional MSF algorithm [1], the best existing adaptive Lee despeckling algorithm [7], the non-constrained robust spatial filtering (RSF) algorithm from [3], the constrained RSF algorithm from [3], the adaptive spatial filtering (ASF) algorithm from [2], and the proposed here POCS-regularized unified DEDR/FBR algorithm (14). We considered a SAR imaging system operating in a typical uncertain RS imaging scenario. The operational uncertainties were simulated via incorporating random perturbations into the regular SFO and contaminating the data with composite multiplicative and additive noise. In the simulation experiments that we report in this paper, the PSF of the fractional SAR system was modeled by of a Gaussian "bell" function in both directions of the 2-D scene (in particular, of 16 pixel width at 0.5 from its maximum for the 512-by-512 pixel-formatted scene). The composite multiplicative noise was simulated as a realization of the $\chi_{2}^{2}$-distributed random variables with the pixel mean value assigned to the actual degraded scene image pixel that directly obeys the RS speckle model [4], [7]. Such signal-dependent multiplicative image noise dominates the additive noise component in the data in the sense that $N_{\Sigma} \gg N_{0}$, hence the estimate $\hat{N}_{\Sigma}$ performed empirically using the local statistics method [7] was used to adjust the regularization degrees of freedom in the DEDR/FBR-POCS procedure (14). Two scenes (the artificially synthesized and borrowed from the real-world RS imagery [8]) were tested. These scenes are displayed in Figures 1(a) and 1(b), respectively. The qualitative simulation results for six different simulated image formation/reconstruction procedures for the first simulated scene are presented in Fig. 2 and for the second scene in Fig. 3, respectively, as specified in the figure captions. The advantage of the unified DEDR/FBR-POCS technique over the previously proposed conventional MSF, de-speckling without DEDR/FBR enhancement and non-adaptive RSF algorithms is evident from the reported simulations.

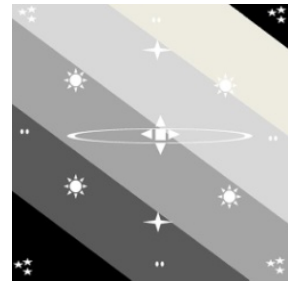

(a)

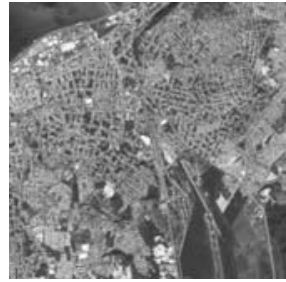

(b)

Fig. 1. Original test scenes: (a) artificially synthesyzed scene; (b) real-world RS scene borrowed from the high-resolution RS imagery [8] 


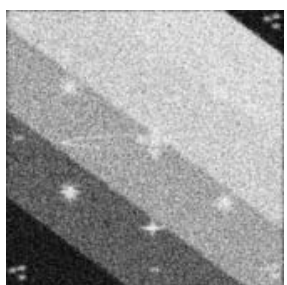

(a)

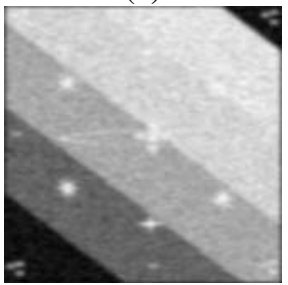

(d)

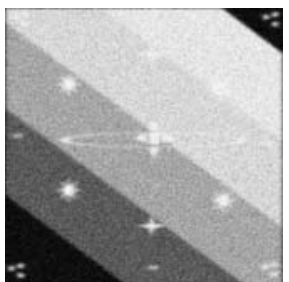

(b)

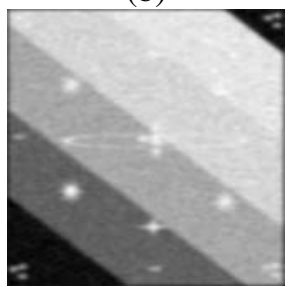

(e)

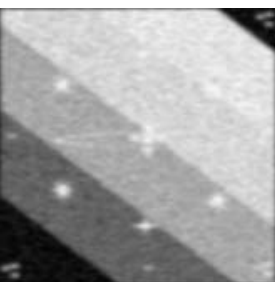

(c)

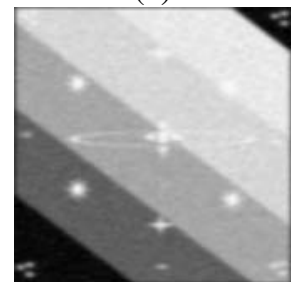

(f)

Fig. 2. Simulation results for the first test scene: (a) degraded SAR scene image formed applying the MSF method [1]; (b) adaptively de-speckled MSF image; (c) image reconstructed applying the non-constrained RSF algorithm [2]; (d) image reconstructed with the constrained RSF algorithm [3]; (e) image reconstructed applying the non-constrained ASF algorithm [2]; (f) image reconstructed applying the developed POCS-regularized DEDR/FBR method

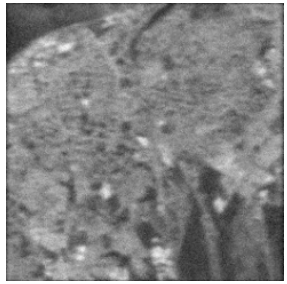

(a)

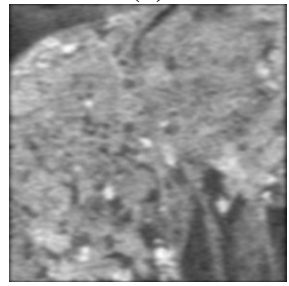

(d)

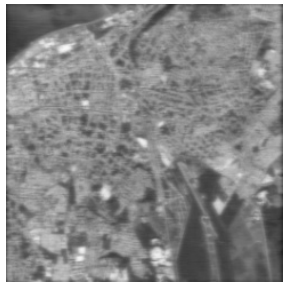

(b)

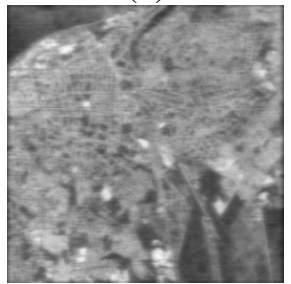

(e)

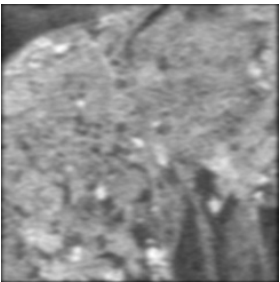

(c)

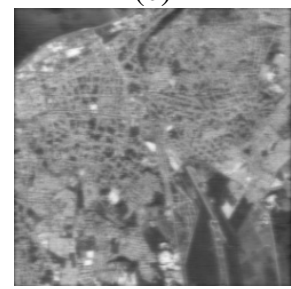

(f)

Fig. 3. Simulation results for the second test scene: (a) degraded SAR scene image formed applying the MSF method [1]; (b) adaptively de-speckled MSF image; (c) image reconstructed applying the non-constrained RSF algorithm [2]; (d) image reconstructed with the constrained RSF algorithm [3]; (e) image reconstructed applying the non-constrained ASF algorithm [3]; (f) image reconstructed applying the developed POCS-regularized DEDR/FBR method 


\section{Concluding Remarks}

In this paper, we have presented the POCS-regularized fixed-point iterative DEDR/FBR method particularly adapted for enhanced RS imaging in the uncertain environment. The unified DEDR/FBR-POCS approach leads to the fixed-point SSP estimator that may be regarded as adaptive post-image-formation enhancement procedure. To facilitate it application for the uncertain imaging scenarios the adaptive scheme for evaluation of the operational degree of freedom (regularization parameter) directly from the uncertain RS data was incorporated into the SSP reconstruction algorithm. We have demonstrated that with such developed adaptive POCSregularized DEDR/FBR technique, the overall RS image enhancement performances can be improved if compared with those obtained using conventional single-look SAR systems that employ auto-focusing techniques or the previously proposed adaptive despeckling and reconstruction filters that do not unify the POCS regularization with the DEDR/FBR method.

\section{References}

1. Shkvarko, Y.: Estimation of wavefield power distribution in the remotely sensed environment: Bayesian maximum entropy approach. IEEE Trans. Signal Proc. 50(9), 2333-2346 (2002)

2. Shkvarko, Y.: Unifying regularization and Bayesian estimation methods for enhanced imaging with remotely sensed data-Part I: Theory. IEEE Trans. Geoscience and Remote Sensing 42(5), 923-931 (2004)

3. Shkvarko, Y., Perez-Meana, H., Castillo-Atoche, A.: Enhanced radar imaging in uncertain environment: A descriptive experiment design regularization approach. Int. J. Navigation and Observation 2008, 1-11 (2008) Article ID 810816

4. Wehner, D.R.: High-Resolution Radar, 2nd edn. Artech House, Boston (1994)

5. Barrett, H.H., Myers, K.J.: Foundations of Image Science. Willey, New York (2004)

6. Ishimary, A.: Wave Propagation and Scattering in Random Media. IEEE Press, NY (1997)

7. Greco, M.S., Gini, F.: Statistical analysis of high-resolution SAR ground clutter data. IEEE Trans. Geoscience and Remote Sensing 45(3), 566-575 (2007)

8. Space Imaging, GeoEye Inc. (2009),

http: / / www. spaceimaging.com/quicklook 